EPJ Web of Conferences 21, 10005 (2012)

DOI: $10.1051 /$ epjconf/20122110005

(C) Owned by the authors, published by EDP Sciences, 2012

\title{
Thermal Neutron Capture onto the Stable Tungsten Isotopes
}

\author{
A. M. Hurst ${ }^{1, a}$, R. B. Firestone ${ }^{1}$, B. W. Sleaford ${ }^{2}$, N. C. Summers ${ }^{2}$, Zs. Revay $^{3}$, L. Szentmiklósi ${ }^{3}$, \\ T. Belgya ${ }^{3}$, M. S. Basunia ${ }^{1}$, R. Capote ${ }^{4}$, H. Choi ${ }^{5}$, D. Dashdorj ${ }^{2,6}$, J. Escher ${ }^{2}$, M. Krticka ${ }^{7}$, and \\ A. Nichols ${ }^{4}$ \\ 1 Lawrence Berkeley National Laboratory, Berkeley, CA 94720, USA \\ 2 Lawrence Livermore National Laboratory, Livermore, CA 94551, USA \\ 3 Institute of Isotopes and Surface Chemistry, H-1525, Budapest, Hungary \\ 4 NAPC-Nuclear Data Section, International Atomic Energy Agency, A-1400 Vienna, Austria \\ 5 Seoul National University, Seoul 151-742, Republic of Korea \\ 6 North Carolina State University, Raleigh, NC 27695, USA \\ 7 Charles University in Prague, Faculty of Mathematics and Physics, CZ-180 00 Prague 8, Czech \\ Republic
}

\begin{abstract}
Thermal neutron-capture measurements of the stable tungsten isotopes have been carried out using the guided thermal-neutron beam at the Budapest Reactor. Prompt singles spectra were collected and analyzed using the HYPERMET $\gamma$-ray analysis software package for the compound tungsten systems ${ }^{183} \mathrm{~W},{ }^{184} \mathrm{~W}$, and ${ }^{187} \mathrm{~W}$, prepared from isotopically-enriched samples of ${ }^{182} \mathrm{~W},{ }^{183} \mathrm{~W}$, and ${ }^{186} \mathrm{~W}$, respectively. These new data provide both confirmation and new insights into the decay schemes and structure of the tungsten isotopes reported in the Evaluated Gamma-ray Activation File based upon previous elemental analysis. The experimental data have also been compared to Monte Carlo simulations of $\gamma$-ray emission following the thermal neutron-capture process using the statistical-decay code DICEBOX. Together, the experimental cross sections and modeledfeeding contribution from the quasi continuum, have been used to determine the total radiative thermal neutron-capture cross sections for the tungsten isotopes and provide improved decay-scheme information for the structural- and neutron-data libraries.
\end{abstract}

\section{Introduction}

Complete neutron-capture $\gamma$-ray spectra are needed for structural- and neutron-data libraries for both applied and basic needs. Improved $\gamma$-ray decay schemes with complete information regarding branching ratios and clarifications over nuclear-structural issues, such as spin-parity $\left(J^{\pi}\right)$ ambiguities of the levels involved, are needed to enhance our basic understanding of the nuclei in question. Furthermore, this information is required in applied areas where a sound nuclear-data library is imperative, e.g. the Monte Carlo Neutron Transport (MCNP) codes for national security applications [1].

This poster describes a method of improving the decay scheme through a comparison of experimental neutron-capture $\gamma$-ray decay data with statistical-model predictions. The experimental decay information is taken from separated and elemental thermal neutron-capture measurements of activated tungsten samples performed at the Budapest Reactor, while the statistical-model calculations have been performed using the Monte Carlo code DICEBOX [2].

\footnotetext{
a e-mail: AMHurst@lbl.gov
}

This is an Open Access article distributed under the terms of the Creative Commons Attribution-Noncommercial License 3.0, which permits unrestricted use, distribution, and reproduction in any noncommercial medium, provided the original work is properly cited. 


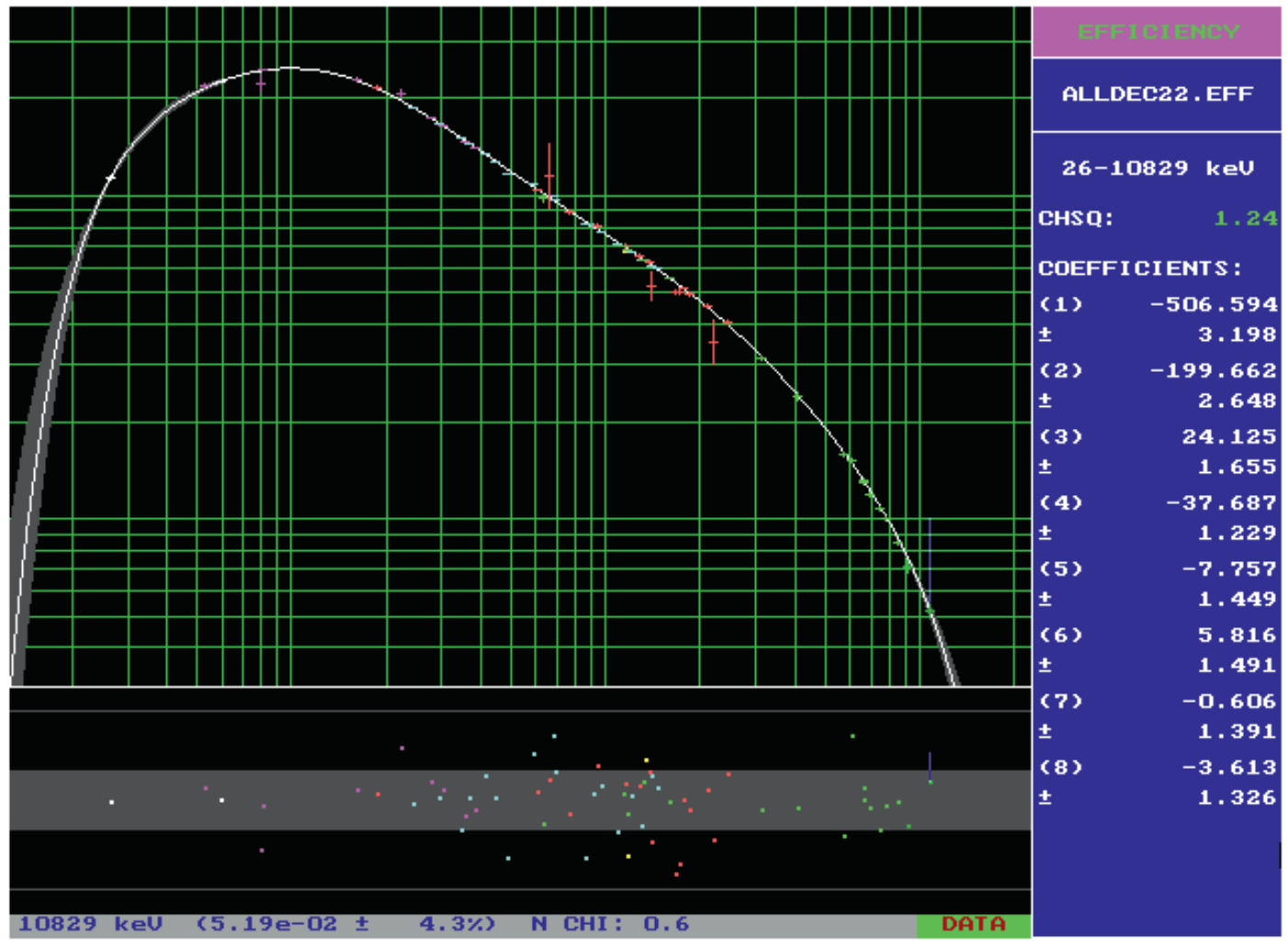

Fig. 1. Relative efficiency curve used in the analysis of the thermal-capture $(\mathrm{n}, \gamma)$ data. The standard radioactive sources: ${ }^{241} \mathrm{Am},{ }^{133} \mathrm{Ba},{ }^{152} \mathrm{Eu},{ }^{207} \mathrm{Bi},{ }^{226} \mathrm{Ra},{ }^{35} \mathrm{Cl}\left[\mathrm{PVC}\right.$ ], and ${ }^{14} \mathrm{~N}$ [Urea], were used and integrated together to cover the energy region of interest from around $50 \mathrm{keV}$ to $10 \mathrm{MeV}$.

\section{Experiment and Data Analysis}

Thermal neutron-capture measurements of stable tungsten isotopes have been performed using a guided thermal-neutron beam, with a flux of $2 \times 10^{6} \mathrm{~cm}^{-2} \cdot \mathrm{s}^{-1}$, at the 10-MW Budapest Reactor [3]. Isotopically-enriched samples ( $\geq 98 \%$ ) of separated tungsten isotopes were prepared as oxide powders $\left(\mathrm{WO}_{3}\right)$ : ${ }^{182} \mathrm{~W} 1261 \mathrm{mg} ;{ }^{183} \mathrm{~W} 400 \mathrm{mg} ;{ }^{186} \mathrm{~W} 200 \mathrm{mg}$. Prompt $\gamma$-ray activation analysis [4] of the corresponding compound tunsgten systems $\left({ }^{183} \mathrm{~W},{ }^{184} \mathrm{~W}\right.$, and ${ }^{187} \mathrm{~W}$, respectively) immediately followed the sample irradiations at a low-background target station located $\sim 30 \mathrm{~m}$ from the shielded reactor wall. The singles capture-(n, $\gamma$ ) data were collected using an $n$-type high-purity germanium (HPGe) detector, $25 \%$ efficient, with closed-end coaxial geometry located $\sim 24 \mathrm{~cm}$ from the target position. The counting efficiency was calibrated using a variety of radioactive sources covering an energy range from around $50 \mathrm{keV}$ to $10 \mathrm{MeV}$. The resulting fit to the effficiency data is shown in Fig. 1. Using this information, the corresponding $(\mathrm{n}, \gamma)$ spectra were then analyzsed with the $\gamma$-ray spectroscopy software package HYPERMET [5]. An example of the fitting procedure to ${ }^{186} \mathrm{~W}(\mathrm{n}, \gamma)$ data (i.e. decay lines corresponding to the ${ }^{187} \mathrm{~W}$ compound) is illustrated in Fig. 2. The isotopic cross sections of the individual $\gamma$ rays $\left(\sigma_{\gamma}\right)$ were deduced relative to strong, well-known $\gamma$ lines reported in each isotope [4]. This standardization procedure is explained in Ref. [6]. In addition to the separated-isotope measurements, the ${ }^{185} \mathrm{~W}$ compound was analyzed from a $200-\mathrm{mg}$ elemental sample comprising natural $\mathrm{WO}_{2}$. Adopting the same standardization procedure, the ${ }^{185} \mathrm{~W} \sigma_{\gamma}$ values were deduced relative to strong sodium lines from a standardized $(\mathrm{n}, \gamma)$ measurement of sodium tungstate $\left(\mathrm{Na}_{2} \mathrm{WO}_{6}\right)$. 


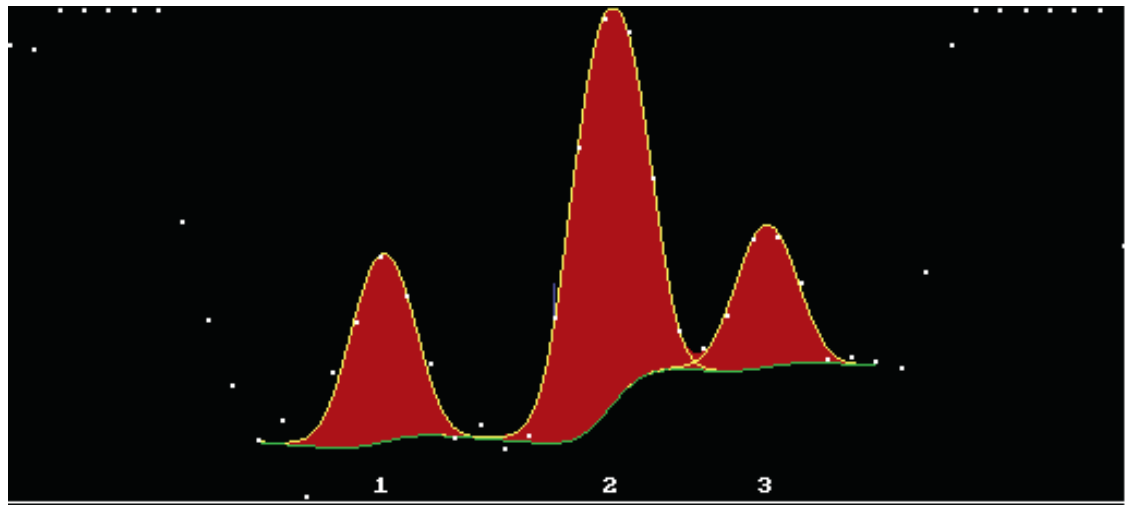

U38W186 . MCA

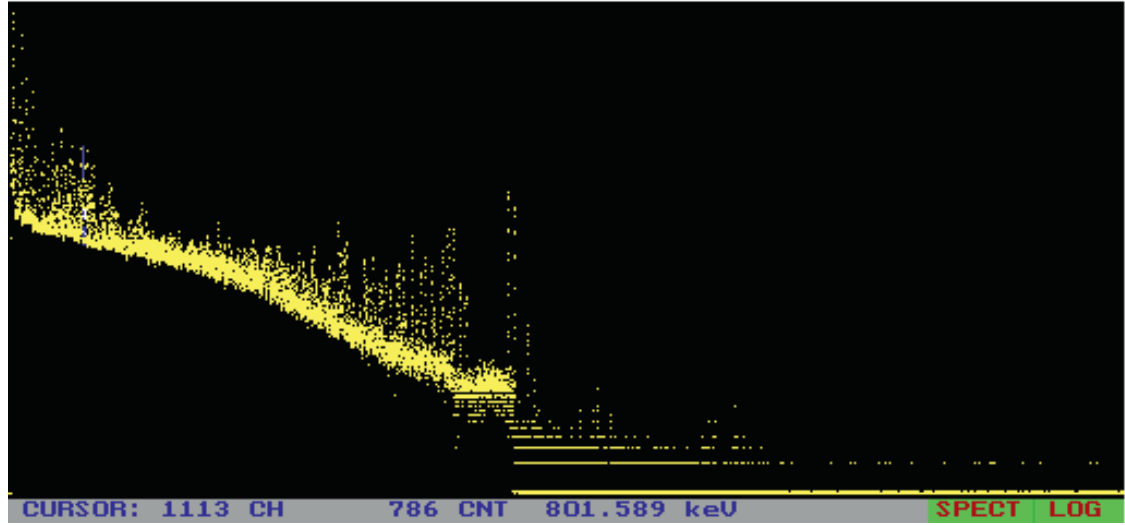

U38W186 . LOG 1101-1126 ch CALI BRAT I ON: Width: Energy: u38w186 . set CORAECT I ONS : ALLDEC22. LIN ALLDEC22 . EFF TRY : 1 CHSQ $(4 * S I G)$ : 0.269827

PEAK(S): 3

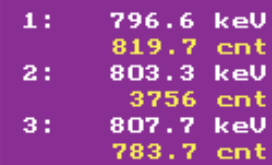

Fig. 2. Prompt ${ }^{186} \mathrm{~W}(\mathrm{n}, \gamma)$ HYPERMET spectrum; $\gamma$-ray transitions at $796.6,803.3$, and $807.7 \mathrm{keV}$ in the ${ }^{187} \mathrm{~W}$ compound are clearly observed.

\section{Statistical Model}

In this work, the Monte Carlo statistical-decay code, DICEBOX [2], has been used to model the thermalcapture $\gamma$ cascade. Based on the experimental $(n, \gamma)$ data collected at the Budapest Reactor, input decks describing the low-lying level schemes of the tungsten isotopes were created for the DICEBOX program. The cut-off energy used in establishing the highest-excitation energy reported in the experimental decay scheme was determined according to the availability of complete information regarding the known $J^{\pi}$ values and $\gamma$-ray branching ratios of the levels involved. This data was supplemented with information regarding unique choices of level density (LD) and photon strength function (PSF) and their appropriate parametrizations. In this analysis, the Back-Shifted Fermi Gas LD with von Egidy parametrization [7] was used in conjunction with a type of generalized Lorentzian PSF for the Giant Dipole Electric Resonance with a parametrization based on the Reference Input Parameter Library (RIPL-3) [8], a PSF for the Giant Dipole Magnetic Resonance based on scissors and spin-flip transition modes, and finally, an isovector-isoscalar model for the Giant Quadrupole Electric Resonance. DICEBOX then uses this information to predict simulated populations to the levels described in the input deck through primary (transitions direct from the neutron-capture state) and secondary $\gamma$-ray transitions. In addition, a feeding contribution from the quasi continuum is also estimated by the code. Since the quasi continuum is an experimentally unresolved region of high level density beneath the capture state, all levels and $\gamma$ rays in this region are randomly generated by DICEBOX according to the choice of LD and PSF in the input deck. The calculation then proceeds using the Monte Carlo method by randomly generating $5 \times 10^{4}$ capture-state $\gamma$-ray decay cascades over ten separate nuclear realizations (level scheme simulations) to generate good statistical variation in the simulated level feedings. 
EPJ Web of Conferences

\section{Outlook}

Evaluations for the compound tungsten isotopes: ${ }^{183} \mathrm{~W},{ }^{184} \mathrm{~W},{ }^{185} \mathrm{~W}$, and ${ }^{187} \mathrm{~W}$, are currently being finalized. In addition to providing structural clarity of the decay schemes for the isotopes being investigated, this work also provides an independent measurement of the total radiative thermal neutron-capture cross section which can be compared to the adopted values in the published literature [9].

\section{References}

1. B. W. Sleaford et al., Capture Gamma-Ray Libraries for Nuclear Applications, Poster contribution to the Third International Workshop on Compound Nuclear Reactions and Related Topics (2011) Budapest, Hungary.

2. F. Becvar, Nucl. Instrum. Methods Phys. Res. A 417, (1998) 434.

3. T. Belgya et al., Ninth International Symposium on Capture Gamma-Ray Spectroscopy and Related Topics, Ed. G. L. Molnár (1997) Budapest, Hungary.

4. Handbook of Prompt Gamma Activation Analysis (Kluwer Academic Publishers, The Netherlands 2004), Ed. G. L. Molnár.

5. Hypermet-PC Version 5.01 (v512), Institute of Isotopes (1995-97), Budapest, Hungary, http://www.iki.kfki.hu/nuclear/hypc/.

6. Zs. Revay and G. L. Molnár, Radiochim. Acta 91, (2003) 361.

7. T. von Egidy and D. Bucurescu, Phys. Rev. C 72, (2005) 044311.

8. R. Capote et al., RIPL - Reference Input Parameter Library for Calculations of Nuclear Reactions and Nuclear Data Evaluations, Nucl. Data Sheets 110, (2009) 3107.

9. S. F. Muhghabghab, Atlas of Neutron Resonances: Resonance Parameters and Thermal Cross Sections $Z=1-100$ (2006), 5th Edition, Elsevier BV, New York. 